\title{
A Fast Inter Mode Decision Algorithm for HEVC Encode System based on Spatial and Temporal Correlations
}

\author{
Yongshuang Yang, Wei An, and Qiuwen Zhang* \\ College of Computer and Communication Engineering, Zhengzhou University of Light Industry, Zhengzhou, 450002, China
}

\begin{abstract}
With the development of video display technology, high efficiency video coding (HEVC) has recently been proposed to optimize the coding efficiency of video encoders, and it has demonstrated great improvements in coding efficiency by using the layered structure of the coding unit (CU), transform unit (TU), and prediction unit (PU). For the purpose of accomplishing the prefect coding efficiency, we must find the best combination of $\mathrm{CU}$, TU, and PU in the situation of the lowest rate distortion (RD) cost, which is time-consuming. Among these CU, TU, and PU, the determination of CU size has the most significant impact on the RD optimization (RDO) of HEVC encoding, which results in a large amount of calculation costs in the operation of PU and TU size determination. Many research works focus on reducing the complexity through fast CU dividing and early skip in the stage of intra-slice coding. We propose a fast inter mode decision algorithm for the HEVC encode system in this paper, and a reformed early CU SKIP detection method constitutes this algorithm. For the current CU block that is being encoded, we use the related spatial coding parameters of CU to evaluate the texture complexity (TC) affecting the CU blocks that are being encoded. In addition, we utilize motion vectors, TU size, and block marking information to gauge the time complication of CU partitions. The proposed method efficiently uses the spatial coding parameters without additional computations. The proposed techniques reduce the encoding time significantly, by up to $47.9 \%$, with an insignificant BD rate increase of about $1.6 \%$.
\end{abstract}

Keywords: HEVC; mode decision; computational complexity; CU split decision

(Submitted on October 3, 2019; Revised on November 10, 2019; Accepted on November 17, 2019)

(C) 2019 Totem Publisher, Inc. All rights reserved.

\section{Introduction}

High efficiency video coding (HEVC) [1] achieves advanced coding performance compared with its previous coding standard H.264, which achieved almost a 50\% BD reduction rate with similar coding efficiency. In addition, with the development of information technology and visual enhancement technology, consumers and research institutions increasingly focus on efficient visual systems. HEVC achieves good performance but introduces huge computational complexity to pattern decision. Therefore, according to the characteristics of the encoding process, it is difficult to save the encoding time of coding blocks (CBs). A fast algorithm for real-time applications of HEVC code is proposed in this paper. Compared with the original HEVC coder, it has considerable enhancements.

HEVC introduces new tools that have multiple block partitioning patterns extended from the code tree unit (CTU), which are different from previous standards. Moreover, these tools inherit from the previous H.264 macroblock (MB). In addition, the CTU coding method has more free size selection for coding tree blocks (CTB). These CTBs are composed of a set of corresponding flexible and differently-sized coding blocks. Encoders perform RD optimization on all potential partitioning pattern groups of CBs to obtain the minimum RD cost representing the best collaboration. The best combination group of CBs is encoded as a video bit stream for later transmission in HEVC.

We propose a fast inter mode decision algorithm in this paper. It consists of two sub-algorithms, an early skip mode detection algorithm for CU, and a fast inter mode decision algorithm. The early CU skip algorithm effectively utilizes the spatial and temporal encoding parameters available in the HEVC coding process, and it does not need feature extraction for

* Corresponding author.

E-mail address: zhangqwen@126.com 
computing costs of fast mode decisions. The proposed scheme utilizes the size of the motion vector (MV), values of the sample-adaptive-offset (SAO) parameter, coded block flag (CBF) data, and TU size to make fast inter mode decisions, such as spatial and temporal coding parameters. Since the process of CU splitting achieves RDO reliance on the TC and motion features, we can use the value of the SAO parameter of the reference block to evaluate the TC. We use the MVs of the current PU and the CBF information to analyze the motion characteristics. The fast inter mode decision algorithm utilizes the correlated tendency of the current PU and its sub-layer PUs to reduce the number of executions of the advanced motion vector prediction (AMVP) process. Consequently, the proposed two sub-algorithms can be easily combined to reduce the coding time. However, we also need to focus on how to eliminate the effects of the corresponding coding efficiency penalties. In this regard, as we study this fast mode decision method, we also combine and compare our method with the methods that have been published.

The rest of this paper is presented as follows. Some related works are given in Section 2. The proposed method is fully introduced in Section 3. The experimental results are presented in Section 4. Finally, the work is concluded in Section 5.

\section{Related Work}

As the HEVC coding tools rapidly develop, many studies have been introduced to decrease the complexity of the HEVC encoder. During the standardization of HEVC [2-3], several fast encoding algorithms were utilized in the HEVC test model (HM). In [4], a fast encoding CU partitioning algorithm in intra prediction was proposed. It has a slight loss in the process of RD cost calculation and achieves significant savings in computational complexity. A fast CU selection method which has pyramid motion divergence was presented in [5], where CU splitting was correlated with motion divergence. In [6], a novel algorithm was presented based on the orientation detection, which uses the local directional variance along some predefined lines. With the research on the adaptive threshold for RD cost, a fast CU measurement decision method in [7] was introduced to decrease the complexity that is in the inter-mode decision. In [8], a fast edge detection scheme in video frame pre-processing was proposed, which helps to reduce the computational complexity and employs intra prediction of HEVC. A hierarchical complexity allocation method that is based on liner programming was proposed in [9] to assign the computational complexity in the frames. Fast CU that is split and pruned for suboptimal CU dividing in HEVC intra coding was introduced in [10]. A fast inter CU decision method was introduced in [11] to discuss the motion correlations between neighbouring CUs. A fast scheme for the CU dividing process of the HEVC encoder, which utilizes machine learning ways, was incorporated in [12]. A novel fast CU decision of measurement and mode method was introduced in [13], and it utilizes the intra forecast of HEVC. A fast CU dividing scheme was employed in [14] for the intra-only (all intra) configuration. In [15], a fast mode decision method that includes adaptive threshold determination was proposed. The introduced scheme that effectively exploits spatial correlation and coding block flag information changed the examination order in [16].

The above algorithms were developed to save time or reduce computational complexity in HEVC. However, most of these fast algorithms pay close attention to the process of $\mathrm{CB}$ encoding or CU segmentation, while the detection of CBs in sequences is not emphasized before the encoding process. In addition, we should make full use of the moving area containing massive CBs, which can be coded as the skip mode. Therefore, there is still potential to reduce the computational complexity of pattern determination for $\mathrm{CB}$ detection.

\section{Proposed Work}

\subsection{Analysis of Spatial Encoding Parameters}

In addition to block filters, HEVC uses a new loop filter, called the SAO filter, as a deblocking filter. The SAO filter uses the method of adding offset values to every pixel to reduce pixel distortion. There are two methods of SAO filtering execution: edge-offset comp and band-offset comp. Edge-offset comp repairs the distortion that is between the initial and reconstructed pixels by subjoining the offset values to the reconstructed pixel. Band-offset comp reduces the distortion between the initial and reconstructed pixels by subjoining the quantized band values to the reconstructed pixel. During the HEVC coding process, SAO filtering will make a decision in the sense of RDO to select an edge offset compensation or a band offset compensation.

Figure 1 displays the different categories of edge types and SAO filters for the edge-offset comp, and it shows how histogram bands values compensate the band-offset. In Figure 1(a), there are four one-dimensional plans that show the four edge categories: the $0^{\circ}, 90^{\circ}, 135^{\circ}$, and $45^{\circ}$ directions. Using the four edge categories, the pixel at position $\mathrm{b}$ can be categorized as one of the above-mentioned categories by comparing it with the values of position a and position c. In other words, if the pixel value of $b$ is less (or greater) than the two adjacent pixel values that correspond to position a and position $\mathrm{c}$, it can be classified as category 1 or category 4 . If the pixel value of $\mathrm{b}$ is equal to the two adjacent pixel values and greater 
than (or less than) the other, this situation can be classified as category 2 or category 3. For all the CUs in CTB, every deblocking filter pixel at the position of $\mathrm{c}$ using the above four edge category templates can be classified into one of them. In the CTB, all positions' pixels can be grouped according to the edge category, and every edge category calculates the average value of the offsetting. For the four edge categories, we can select the best edge type in the sense of RDO to acquire all the distortions that are caused by four offsettings. Finally, the selected edge type and its offsetting values will be written into the bit stream.

Figure 1(c) shows two groups of band-offset. According to the offset compensation value, the CTB's pixels can be quantized into 32 groups. The encoder only sends first band position information and average offset values of the four consecutive bands to the encoder to repair the distortion between the initial and rebuild pixels. However, in our TC research, we do not use offset compensation, since it does not transport the message of texture. In our proposed fast algorithm, it should be noted that the information of the edge category has been procured during the SAO process. Because every pixel in the CTB can be classified as one of the four edge categories, a $4 \times \log 22 N \times 2 N$ buffer is needed to save the information of edge categories for each CTU.

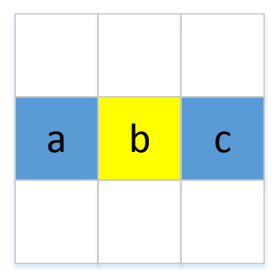

Catgory1

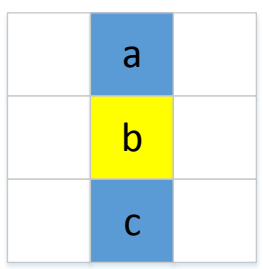

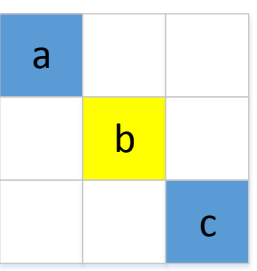

Catgory3

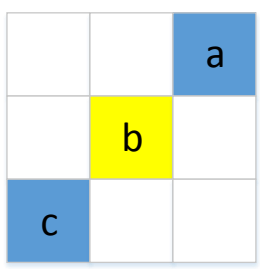

Catgory4

\section{更}
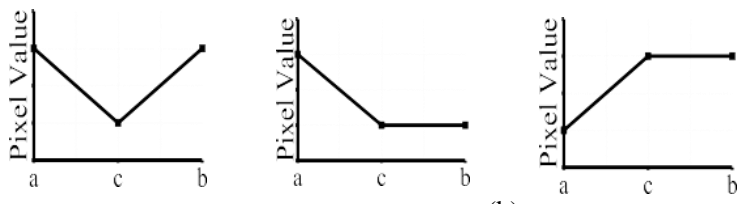

(b)
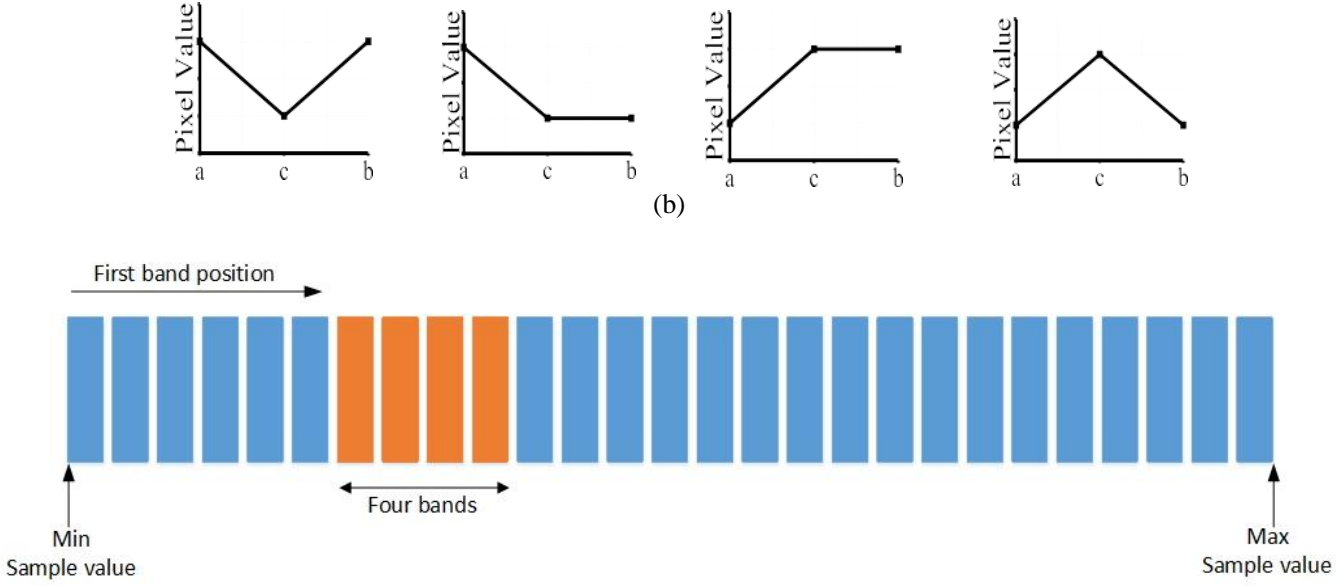

(c)

Figure 1. Edge type and category for compensation of edge-offset, and column diagram bands for band-offset compensation: (a) edge type, (b) edge category, (c) band offset compensation

\subsection{Analysis of HEVC Mode Decision Process}

HEVC permits flexibility because it uses the layeral block construction of CU, TU, and PU. CU is the basic encoding unit and has a variable size hierarchical quadtree structure with a range from $64 \times 64$ to $8 \times 8$. PU is the basic unit for inter- and intra-frame prediction. TU is the basic unit of transformation and quantization. Non-square PU is supported in the interframe prediction mode. A picture can be divided into many slices. In other words, the slice is a constituent element of a whole picture. Then, slices are divided into coding tree units (CTUs), which are the largest size of CU $(64 \times 64)$. A CTU can be divided into four of the same CUs, and each of the CUs can be further split into four CUs by iterations unless reaches the smallest $\mathrm{CU}$ size $(8 \times 8$ size of pixel) is reached. The PU decision process is shown in Figure 2 . The process involves finding the partition size and prediction mode. In the flowchart, $\mathrm{nN} \times \mathrm{nN}$ indicates a series of inter mode PU sizes, such as $N$ $\times 2 N, 2 N \times N, N \times N$, and asymmetric sub-partitions. Next, every CU will be encoded in the inter mode or intra mode, and the PU partition will be determined in the processing of the PU decision. The AMVP mode or merge mode can be adopted for the PU. The concept of merge mode is equivalent to the skip mode and the direct mode in the standard of H.264/AVC, where the current PU that is specified inherits reference image indexes and motion vectors from the adjacent PU. Therefore, 
there is no MV, and the indexes of the reference image are transmitted. When the PU size is $2 N \times 2 N$, it indicates a special situation in the skip mode, and there is no texture information sent to the decoder. The AMVP process is most complex during the encoding process; it not only calculates the RD cost for every PU but also analyzes the motion estimation to find the PU's optimal mode. In order to reduce the computation complexity of this process, we should reduce the unnecessary AMVP process.

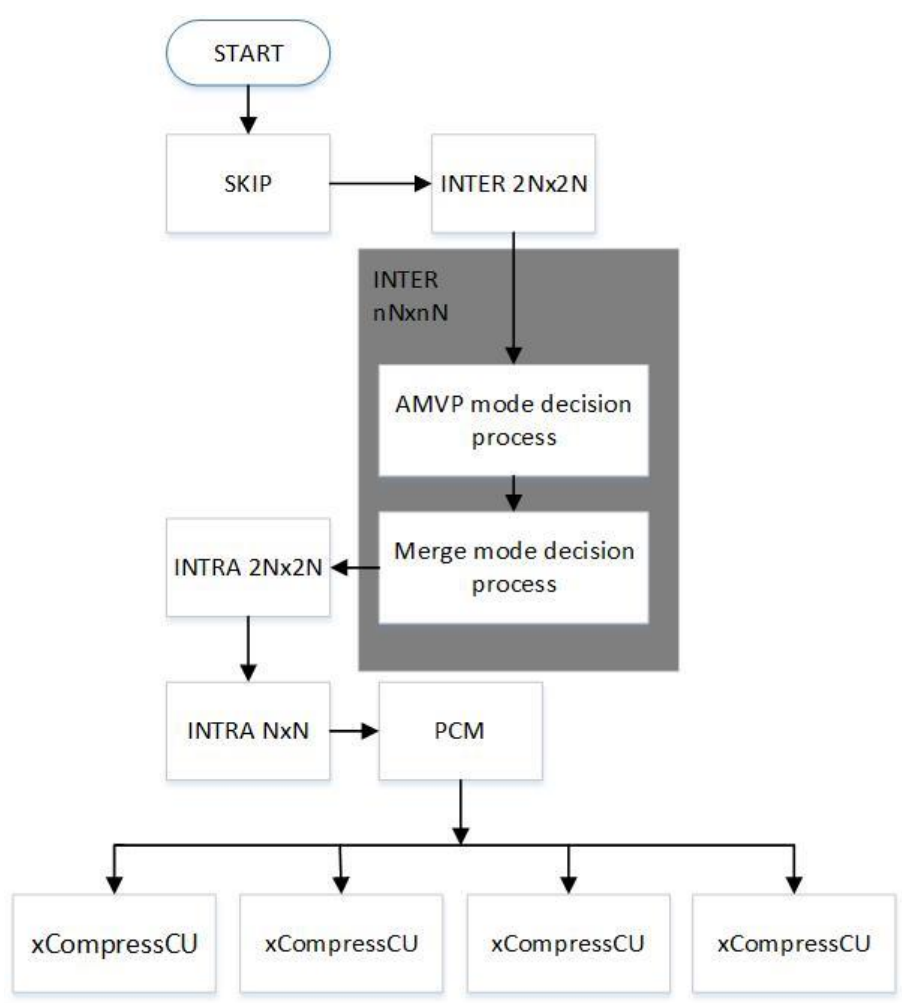

Figure 2. Flowchart of mode decision (in HM 12.0)

\subsection{Improved Early Skip Mode based on CU's TC}

When considering the use of CU for encoding, we first check the skip mode of CU through our early skip mode method.

SKIP detection algorithms have already been proposed in HEVC. In particular, most proposed methods depend on comparing the $\mathrm{RD}$ cost and the previous average $\mathrm{RD}$ cost. The proposed method can be summarized as

$$
\begin{gathered}
\text { SKIP } \\
F_{\text {SKIP }}^{C U_{n}} \underset{\text { c }}{<} \\
\text { non }- \text { SKIP }
\end{gathered}
$$

$F_{S K I P}^{C U_{n}}$ represents the current block's RD cost; $\alpha$ is the weight factor, the value of which is set to $1.6 ; F_{S K I P}^{C U_{i}}$ is the $k^{\text {th }} \mathrm{CU}$ to be encoded with skip mode; and $N$ is the amount of the CUs. The fast encoding process will be adopted, and no other encoding mode will be attempted when the best encoding mode of CU is determined to be the skip mode.

The value of the complex texture CU of the RD cost is greater than the simple one. We can expect to execute the skip mode when considering the CU block's TC. The information of the edge category in SAO parameters is helpful to decide the SKIP mode. Based on the useful information of the edge category, we can modify Equation (1) as follows:

$$
F_{S K I P}^{C U_{n}^{T C_{k}}}<\alpha \frac{1}{N_{T C_{k}}} \sum_{i=1}^{N_{T C_{k}}} F_{S K I P}^{C U_{i}^{T C_{k}}}
$$


$F_{S K I P}^{C U_{n}^{T C_{j}}}$ is the value of the RD cost, and $C U_{n}^{T C_{k}}$ is part of the $k^{\text {th }} \mathrm{TC}$ class. If CU is a complex texture class, $j=0$. The weight factor $\alpha$ is set to 1.2 to avoid high error detection.

\subsection{Improved Fast Inter Mode Decision Method}

When the PU size is $2 N \times 2 N$, the PU in the sublayer has similar features. Table 1 illustrates the correlation of the optimal inter mode of the $2 N \times 2 N$ PU and the optimal mode of PUs in the sublayer. The optimal mode of every inter-frame mode of the $n N \times n N$ PU in the sublayer can be defined as the mode of the father-layer PU. As shown in Table 1, the probability is about $96 \%$ when the best mode of $2 N \times 2 N$ frames is chosen as the merge or skip mode. The best mode of the sublayer is more likely to be selected as the merge mode. Therefore, in this case, we can avoid performing the AMVP procedure.

\begin{tabular}{|c|c|c|c|} 
Table 1. Probability of the best inter-modes of $n N \times n N$ in sequences \\
\hline Size & $P(M \mid C=M)$ & $P(M \mid C=S)$ & $P(M \mid C=A)$ \\
\hline $\begin{array}{c}\text { Traffic } \\
(2560 \times 1600)\end{array}$ & 0.68 & 0.2 & 0.53 \\
\hline $\begin{array}{c}\text { Parry Scene } \\
(1920 \times 1080)\end{array}$ & 0.77 & 0.97 & 0.42 \\
\hline $\begin{array}{c}\text { Blowing Bubbles } \\
(832 \times 480)\end{array}$ & 0.81 & 0.97 & 0.38 \\
\hline Average & 0.74 & 0.96 & 0.46 \\
\hline
\end{tabular}

* $M$ (merge), $S$ (skip), $A$ (AMVP).

Based on the above analysis of observation, when the best mode of the selected Inter $2 N \times 2 N$ PU frames is the skip mode, we propose the method that does not execute the AMVP process for the sublayer of the current CU.

Our fast procedure can be expressed as

$$
\text { AMVP in } n N \times n N=\left\{\begin{array}{l}
\text { no check, if Inter } 2 N \times 2 N=S K I P \\
\text { check, }
\end{array}\right.
$$

When it is satisfied with the "no check" condition in (3), the encoder will execute the proposed fast method without performing the unnecessary AMVP process.

\subsection{Overall Algorithm}

Figure 3 shows the flow chart of our proposed method. After encoding $2 N \times 2 N$ PU, the process that is detected for an early CU skip mode is performed on the present CU, as described in Section 3.3. Then, the obtained time coding parameters will be obtained (e.g. TU, CBF data, and MV).

- If the best candidate mode is the SKIP mode, and condition (1) or (2) is not satisfied, the flow will enter the early skip mode.

- If the conditions are not satisfied, it will enter the sub-algorithm proposed in Section 3.4. Determine condition (3); if it is satisfied, the encoder will not execute the AMVP process for the current PU's all sub-partitions.

\section{Experimental Results}

\subsection{Experimental Conditions}

The proposed method is performed on the HM12.0 test mode. The QPs values are 22, 27, 32, and 37. The frames of video sequences are divided into $G O P=8$, and sequences are tested under both random access (RA) and low delay (LB) test conditions. We use the HEVC software model HM12.0 to test and verify the experiment. The encoding computational complexity reduction is computed using time saving (TS) according to the following equation:

$$
T S[\%]=\frac{\text { Enc.time }(\mathrm{HM} 12.0)-\text { Enc.time }(\text { Proposed })}{\text { Enc.time }(\mathrm{HM} 12.0)} \times 100 \%
$$




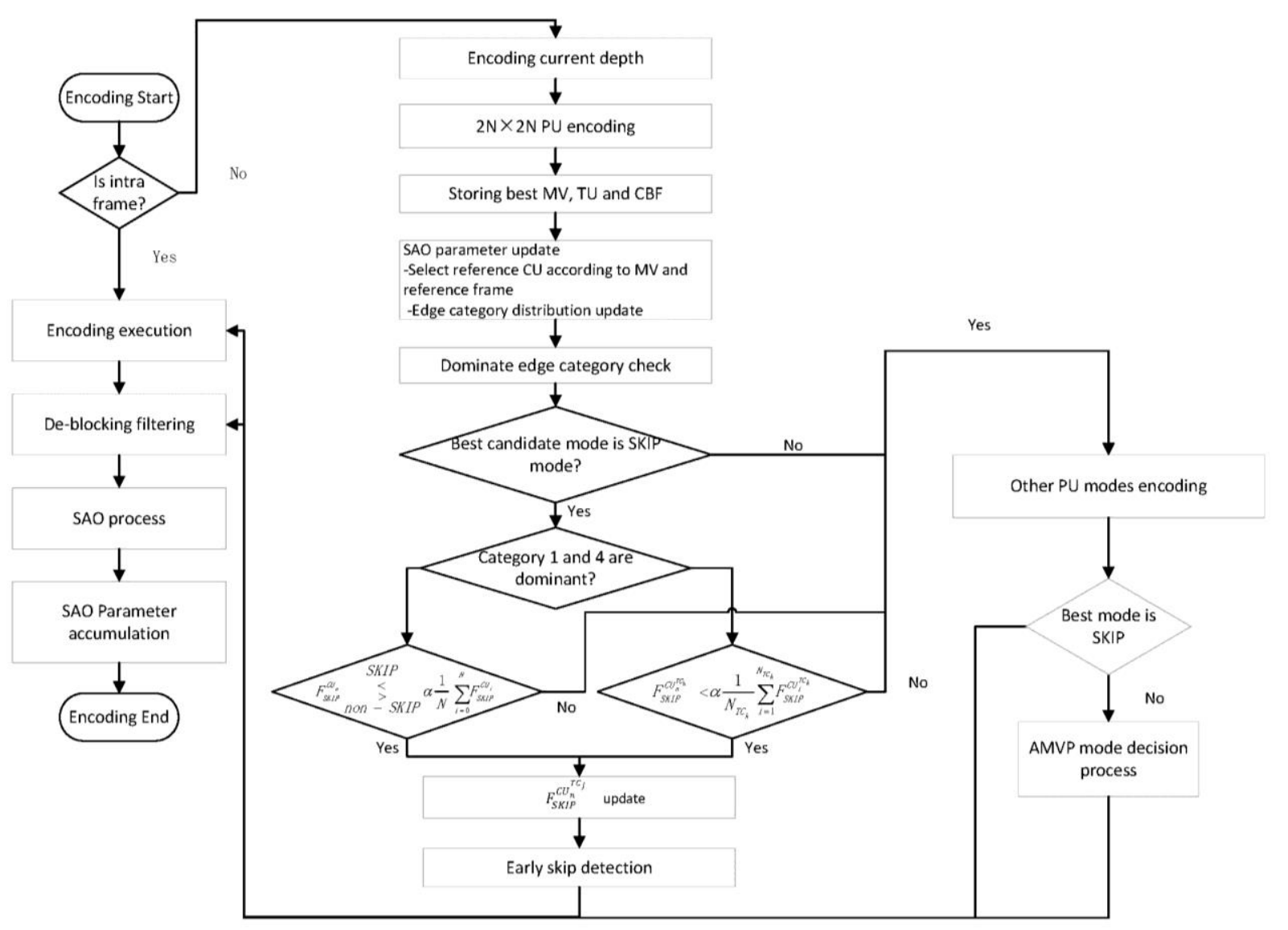

Figure 3. The overall fast inter encoding scheme

\subsection{Evaluation of the Proposed Method}

Table 2 shows the experiment results. To investigate the contributions of the two independent sub-algorithms from Sections 3.3 and 3.4, we study the independent and combined methods. Compared with HM12.0, the methods from Sections 3.3 and 3.4 reduce the encoding time remarkably. The proposed method under the RA condition reduces the encoding time by $47.95 \%$, while causing a negligible loss of bit-rate (with a $B D B R$ increase of about $1.6 \%$ ).

Table 2. Experimental results (single method and combined method under RA condition)

\begin{tabular}{|c|c|c|c|c|c|c|c|}
\hline \multirow{3}{*}{ Size } & \multirow{3}{*}{ Sequences } & \multirow{2}{*}{\multicolumn{2}{|c|}{ Early skip mode only (Section 3.3) }} & \multirow{2}{*}{\multicolumn{2}{|c|}{ Fast inter mode decision only (Section 3.4) }} & \multirow{2}{*}{\multicolumn{2}{|c|}{$\frac{\text { Overall performance }}{\text { (Sections 3.3-3.4) }}$}} \\
\hline & & & & & & & \\
\hline & & $B D B R(\%)$ & $T S(\%)$ & $B D B R(\%)$ & $T S(\%)$ & $B D B R(\%)$ & $T S(\%)$ \\
\hline $\begin{array}{c}\text { Class A } \\
(2560 \times 1600)\end{array}$ & PeopleOnStreet & 1.7 & 13.8 & 0.8 & 22.7 & 0.9 & 26.9 \\
\hline \multirow{4}{*}{$\begin{array}{c}\text { Class B } \\
(1920 \times 1080)\end{array}$} & Kimonol & 0.8 & 29.9 & 0.3 & 34.2 & 1.3 & 58.2 \\
\hline & Cactus & 1.2 & 30.0 & 0.8 & 34.6 & 2.8 & 56.8 \\
\hline & BasketballDrive & 1.5 & 25.9 & 0.7 & 32.7 & 2.0 & 50.9 \\
\hline & BQTerrace & 0.8 & 21.4 & 0.3 & 38.1 & 1.6 & 54.5 \\
\hline \multirow{3}{*}{$\begin{array}{c}\text { Class C } \\
(832 \times 480)\end{array}$} & BasketballDrill & 1.4 & 32.8 & 0.7 & 29.1 & 1.9 & 45.2 \\
\hline & BQMall & 1.6 & 37.1 & 0.5 & 34.1 & 2.2 & 48.6 \\
\hline & PartyScene & 1.2 & 29.9 & 0.4 & 28.8 & 0.8 & 37.7 \\
\hline \multicolumn{2}{|c|}{ Average } & 1.2 & 26.6 & 0.6 & 32.7 & 1.6 & 47.9 \\
\hline
\end{tabular}


To validate the reduction in computational complexity, we compare our method with the proposed methods [4] and [5] under the RA condition and LD condition. Table 3 shows the results of the experiment. When our proposed method in Section 3.3 is combined with the method in Section 3.4, the total encoding time decreases by $40.1 \%$ and the BD rate increases by $1.5 \%$ under the RA condition. The total encoding time decreases by $47.9 \%$ and the BD rate increases by $1.6 \%$ under the LD condition. Compared with the proposed method in [4], the average encoding time decreases by $41.4 \%$ and the BD rate increases by $1.1 \%$ under the RA condition. There is a $44.8 \%$ decrease in time and a $1.2 \%$ increase in the BD rate under the LD condition. The early CU termination method, which was proposed in [5], results in a $28.6 \%$ encoding time reduction with a $1.7 \%$ BD rate increase under the RA condition. Meanwhile, the time decreases by $31.4 \%$ and the $\mathrm{BD}$ rate increases by $2.4 \%$ under the LD condition. The experiment demonstrates the efficiency of our fast inter mode decision method, which utilizes spatial and temporal parameters in video encoding, and it performs better than the latest methods of HEVC.

Table 3. Experimental results (proposed scheme with recent work under RA condition and LD condition)

\begin{tabular}{|c|c|c|c|c|c|c|c|c|}
\hline \multirow{2}{*}{ Config. } & \multirow{2}{*}{ Size } & \multirow{2}{*}{ Sequences } & \multicolumn{2}{|c|}{$\begin{array}{l}\text { Proposed scheme } \\
\text { (Sections 3.3-3.4) }\end{array}$} & \multicolumn{2}{|c|}{$\begin{array}{c}\text { J.Kim's early skip detection method } \\
\text { [4] implemented in HM } 12.0\end{array}$} & \multicolumn{2}{|c|}{$\begin{array}{l}\text { K.Chou's early CU termination } \\
\text { method [5] implemented in HM } 12.0\end{array}$} \\
\hline & & & $\begin{array}{c}B D B R \\
(\%)\end{array}$ & $T S(\%)$ & $B D B R(\%)$ & $T S(\%)$ & $B D B R(\%)$ & $T S(\%)$ \\
\hline \multirow{12}{*}{$\begin{array}{l}\text { Random } \\
\text { access }\end{array}$} & \multirow{2}{*}{$\begin{array}{c}\text { Class A } \\
(2560 \times 1600)\end{array}$} & Traffic & 2.0 & 41.7 & 1.0 & 54.7 & 2.0 & 41.3 \\
\hline & & PeopleOnStreet & 1.5 & 28.0 & 0.4 & 32.5 & 2.2 & 19.9 \\
\hline & \multirow{5}{*}{$\begin{array}{c}\text { Class B } \\
(1920 \times 1080)\end{array}$} & Kimonol & 0.8 & 47.9 & 0.8 & 38.0 & 0.3 & 27.8 \\
\hline & & ParkScene & 1.1 & 48.3 & 0.8 & 40.5 & 0.9 & 28.9 \\
\hline & & Cactus & 2.2 & 48.0 & 0.5 & 43.5 & 3.1 & 36.4 \\
\hline & & $\begin{array}{l}\text { BasketballDriv } \\
\mathrm{e}\end{array}$ & 1.2 & 42.6 & 0.8 & 42.3 & 1.0 & 25.7 \\
\hline & & BQTerrace & 3.0 & 47.5 & 1.3 & 42.7 & 1.8 & 32.2 \\
\hline & \multirow{4}{*}{$\begin{array}{c}\text { Class C } \\
(832 \times 480)\end{array}$} & BasketballDrill & 2.0 & 37.0 & 1.9 & 44.0 & 1.3 & 38.5 \\
\hline & & BQMall & 1.2 & 40.9 & 2.0 & 43.5 & 2.2 & 25.3 \\
\hline & & PartyScene & 0.4 & 33.0 & 1.0 & 40.0 & 3.3 & 25.1 \\
\hline & & RaceHorses & 1.1 & 26.1 & 1.0 & 34.1 & 1.1 & 14.0 \\
\hline & \multicolumn{2}{|c|}{ Average } & 1.5 & 40.1 & 1.0 & 41.4 & 1.7 & 28.6 \\
\hline \multirow{12}{*}{$\begin{array}{l}\text { Low } \\
\text { delay }\end{array}$} & \multirow{2}{*}{$\begin{array}{c}\text { Class A } \\
(2560 \times 1600)\end{array}$} & Traffic & 0.8 & 61.6 & 1.1 & 60.5 & 2.1 & 44.1 \\
\hline & & PeopleOnStreet & 0.9 & 26.9 & 0.2 & 42.5 & 4.0 & 23.3 \\
\hline & \multirow{5}{*}{$\begin{array}{c}\text { Class B } \\
(1920 \times 1080)\end{array}$} & Kimonol & 1.3 & 58.2 & 1.0 & 47.3 & 0.4 & 31.2 \\
\hline & & ParkScene & 1.2 & 52.6 & 0.9 & 45.2 & 1.0 & 33.7 \\
\hline & & Cactus & 2.8 & 56.8 & 1.0 & 42.1 & 3.2 & 40.6 \\
\hline & & $\begin{array}{l}\text { BasketballDriv } \\
\mathrm{e}\end{array}$ & 2.0 & 50.9 & 1.0 & 41.8 & 1.4 & 28.7 \\
\hline & & BQTerrace & 1.6 & 54.5 & 1.1 & 49.7 & 1.2 & 35.7 \\
\hline & \multirow{4}{*}{$\begin{array}{c}\text { Class C } \\
(832 \times 480)\end{array}$} & BasketballDrill & 1.9 & 45.2 & 2.1 & 39.8 & 5.2 & 34.1 \\
\hline & & BQMall & 2.2 & 48.6 & 1.6 & 40.0 & 2.9 & 28.8 \\
\hline & & PartyScene & 0.8 & 37.7 & 1.1 & 45.8 & 2.5 & 27.0 \\
\hline & & RaceHorses & 2.2 & 33.9 & 1.8 & 38.5 & 2.0 & 17.7 \\
\hline & \multicolumn{2}{|c|}{ Average } & 1.6 & 47.9 & 1.2 & 44.8 & 2.4 & 31.4 \\
\hline
\end{tabular}

\section{Conclusions}

A fast algorithm for inter mode decision algorithm of mode decision in HEVC is proposed in this paper. It utilizes spatial and temporal parameters in video sequences before the encoding process for the skip mode. The spatial encoding parameters of CU blocks clearly correlate with CU encode blocks. It is crucial to analyze the complexity of the MV and TC of the current block. Moreover, it is beneficial to use encoder parameters to make efficient fast CU splitting decisions based on the motion and texture complexity analyzed, and this does not add computation complexity to the coding process. The proposed scheme combines spatial encoding parameters to establish an accurate and effective mode decision process. As a spatial encoding parameter, we use SAO edge parameters to predict the best inter $2 N \times 2 N$ mode and the texture characteristics of each CU block. The remaining PUs in the sublayer will ignore the unnecessary AMVP process if it is the skip mode. Our 
scheme reduces the encoding time with small bit-rate loss. Combined with the existing fast methods, the proposed method can demonstrate excellent performance.

\section{Acknowledgements}

This work was supported in part by the National Natural Science Foundation of China (No. 61771432 and 61302118), Scientific Project (No. 182102210156), Young Key Teacher Project (No. 2016GGJS-087), and Education Department Project (No. 17B510011).

\section{References}

1. G. J. Sullivan, J. M. Boyce, Y. Chen, J. R. Ohm, C. A. Segall, and A. Vetro, "Standardized Extensions of High Efficiency Video Coding (HEVC)," IEEE Journal of Selected Topics in Signal Processing, Vol. 7, No. 6, pp. 1001-1016, December 2013

2. S. -H. Jung and H. W. Park, "A Fast Mode Decision Method in HEVC using Adaptive Ordering of Modes," IEEE Transactions on Circuits and Systems for Video Technology, Vol. 26, No. 10, pp. 1846-1858, October 2016

3. J. Lee, S. Kim, K. Lim, and S. Lee, "A Fast CU Size Decision Algorithm for HEVC," IEEE Transactions on Circuits and Systems for Video Technology, Vol. 25, No. 3, pp. 411-421, March 2015

4. J. Kim, J. Yang, K. Won, and B. Jeon, "Early Determination of Mode Decision for HEVC," IEEE Proceedings on Picture Coding Symposium (PCS2012), pp. 449-452, May 2012

5. K. Choi, S. -H. Park, and E. S. Jang, "Coding Tree Pruning based CU Early Termination,” Document JCTVC-F092, JCT-VC, Torino, Italy, July 2011

6. D. Ruiz, G. Fernández-Escribano, J. L. Martínez, and P. Cuenca, "Fast Intra Mode Decision Algorithm based on Texture Orientation Detection in HEVC," Signal Processing-Image Communication, Vol. 44, pp. 12-28, May 2016

7. S. Ahn, B. Lee, and M. Kim, "A Novel Fast CU Encoding Scheme based on Spatiotemporal Encoding Parameters for HEVC Inter Coding," IEEE Transactions on Circuits and Systems for Video Technology, Vol. 25, No. 3, pp. 422-435, March 2015

8. G. Correa, P. Assuncao, L. Agostini, and L. A. da Silva Cruz, "Complexity-Aware High Efficiency Video Coding," pp. 125-158, Springer, Switzerland, 2016

9. J. H. Lee, K. S. Jang, B. G. Kim, S. Jeong, and J. S. Choi, "Fast Intra Mode Decision Algorithm based on Local Binary Patterns in High Efficiency Video Coding (HEVC)," in Proceedings of 2015 IEEE International Conference on Consumer Electronics (ICCE), March 2015

10. S. Cho and M. Kim, "Fast CU Splitting and Pruning for Suboptimal CU Partitioning in HEVC Intra Coding," IEEE Transactions on Circuits and Systems for Video Technology, Vol. 23, No. 9, pp. 1555-1564, September 2013

11. Z. Xu and B. Min, “A Fast Inter CU Decision Algorithm for HEVC,” Signal Processing: Image Communication, Vol. 60, pp. 211-223, February 2018

12. D. Lee and J. Jeong, "Fast CU Size Decision Algorithm using Machine Learning for HEVC Intra Coding," Signal Processing: Image Communication, Vol. 62, pp. 33-41, March 2018

13. Y. Song, Y. Zeng, and X. Li, "Fast CU Size Decision and Mode Decision Algorithm for Intra Prediction in HEVC," Multimedia Tools and Applications, Vol. 76, No. 2, pp. 2001-2007, January 2017

14. M. Maazouz, N. Batel, N. Bahri, and N. Masmoudi, "Homogeneity-based Fast CU Partitioning Algorithm for HEVC Intra Coding," International Journal Engineering Science and Technology, Vol. 22, No. 3, pp. 706-714, June 2019

15. X. Huang, Q. Zhang, and X. Zhao, "Fast Inter-Prediction Mode Decision Algorithm for HEVC," Signal, Image and Video Processing, Vol. 11, No. 1, pp. 33-40, January 2017

16. S. Hong, D. Yang, and B. Park, "An Efficient Intra-Mode Decision Method for HEVC," Signal, Image and Video Processing, Vol. 10, No. 6, pp. 1055-1063, September 2016 